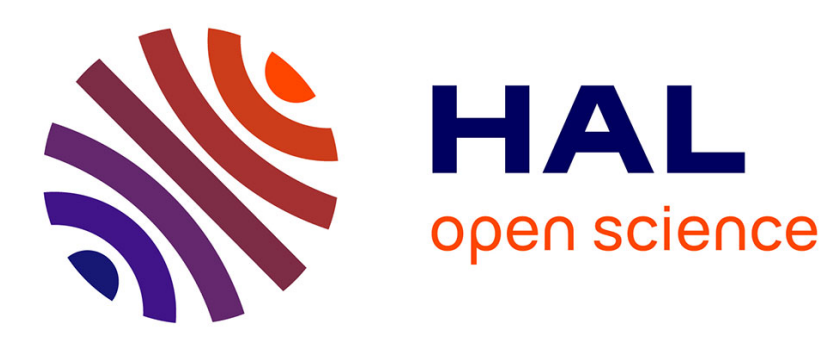

\title{
New methodologies for adaptive sliding mode control
}

Franck Plestan, Yuri Shtessel, Vincent Bregeault, Alexander Poznyak

\section{To cite this version:}

Franck Plestan, Yuri Shtessel, Vincent Bregeault, Alexander Poznyak. New methodologies for adaptive sliding mode control. International Journal of Control, 2010, 83 (9), pp.1907-1919. hal-00626498

\section{HAL Id: hal-00626498 \\ https://hal.science/hal-00626498}

Submitted on 26 Sep 2011

HAL is a multi-disciplinary open access archive for the deposit and dissemination of scientific research documents, whether they are published or not. The documents may come from teaching and research institutions in France or abroad, or from public or private research centers.
L'archive ouverte pluridisciplinaire HAL, est destinée au dépôt et à la diffusion de documents scientifiques de niveau recherche, publiés ou non, émanant des établissements d'enseignement et de recherche français ou étrangers, des laboratoires publics ou privés. 


\title{
New methodologies for adaptive sliding mode control
}

\author{
F. Plestan ${ }^{+*}$ Y. Shtessel ${ }^{++}$, V. Brégeault ${ }^{+}$, A. Poznyak ${ }^{\dagger}$ \\ ${ }^{+}$Ecole Centrale de Nantes-IRCCyN, Nantes, France \\ ${ }^{++}$The University of Alabama in Huntsville, Huntsville, USA \\ ${ }^{\dagger}$ CINVESTAV-IPN, Mexico DC, Mexico \\ Submitted to \\ International Journal of Control - Revised version
}

\begin{abstract}
This paper proposes new methodologies for the design of adaptive sliding mode control. The goal is to obtain a robust sliding mode adaptive gain control law with respect to uncertainties and perturbations without the knowledge of uncertainties/perturbations bound (only the boundness feature is known). The proposed approaches consist in having a dynamical adaptive control gain that establishes a sliding mode in finite time.. Gain dynamics ensures also that there is no over-estimation of the gain with respect to the real a priori unknown value of uncertainties. The efficacy of both proposed algorithms is confirmed on a tutorial example and while controlling an electropneumatic actuator.
\end{abstract}

\section{Introduction}

The sliding mode control is a very popular strategy for control of nonlinear uncertain systems, with a very large frame of applications fields [23, 25]. Due to the use of discontinuous function and high control gain, its main features are the robustness of closed-loop system and the finite-time convergence. However, its design requires the knowledge of uncertainties bound, which could be, by a practical point-of-view, a hard task; it often follows that this bound is over-estimated, which yields to excessive gain. Then, the main drawback of the sliding mode control, the well-known chattering phenomenon (for its analysis, see [3, 4]), is important and could damage actuators and systems. A first way to reduce the chattering is the use of a boundary layer: in this case, many approaches have proposed adequate controller gains tuning [22]. A second way to decrease the chattering phenomenon is the use of higher order sliding mode controller $[14,1,15,16,12,19]$. However, in these both control approaches, knowledge of uncertainties bound is required.

As the objective is the not-requirement of the uncertainties bound, an other way consists in

\footnotetext{
*Corresponding author. E-mail address: Franck.Plestan@irccyn.ec-nantes.fr. This work has been made while Franck Plestan was on sabbatical at UAH. This stay has been financialy supported in part by Ecole Centrale de Nantes, IRCCyN, and Centrale Initiatives Foundation, Lille, France.
} 
using adaptive sliding mode, the goal being to ensure a dynamical adaptation of the control gain in order to be as small as possible whereas sufficient to counteract the uncertainties/perturbations. As recalled previously, this problem is an exciting challenge for applications given that, in many cases, gains are also over-estimated, which gives larger control magnitude and larger chattering. In order to adapt the gain, many controllers based on fuzzy tools $[18,24]$ have been published ; however, these papers do not guarantee the tracking performances. In [10], gain dynamics directly depends on the tracking error (sliding variable): the control gain is increasing since sliding mode is not established. Once it is the case, gain dynamics equals 0 . The main drawback of this approach is the gain over-estimation with respect to uncertainties bound (see for example in [10]-Section III). Furthermore, this approach is not directly applicable, but requires modifications for its application to real systems: thus, the sign function is replaced by a saturation function whom the boundary layer width affects accuracy and robustness. Furthermore, no boundary layer width tuning methodology is provided. A method proposed in [13] in order to limit the switching gain must be mentioned. The idea is based on use of equivalent control: once sliding mode occurs, disturbance magnitude is evaluable and allows an adequate tuning of control gain. However, this approach requires the knowledge of uncertainties/perturbations bounds and the use of low-pass filter, which introduces signal magnitude attenuation, delay, and transient behavior when disturbances are acting. In [9], a gain-adaptation algorithm is proposed by using sliding mode disturbance observer. The main drawback is that the knowledge of uncertainties bounds is required to design observer-based controller.

The objective of the current paper is to propose new methodologies for control of a class of uncertain nonlinear systems for which uncertainties are bounded but this bound (which is finite) is not known. The first controller is based on an gain adaptation law derived from coupling of $[10,13]$ methods, whereas the second one is using an original gain adaptation law. In the both control algorithms, the gain is dynamically tuned in order to ensure the establishment of a sliding mode; once this sliding mode is achieved, the gain is adjusted in order to get a "sufficient" value in order to counteract the perturbations and uncertainties.

Section 2 states the problem, displays some recalls on sliding mode and presents a review of $[10,13]$ by addressing their deficiencies. Section 3 displays the both proposed adaptive sliding mode control algorithms. In order to prove the feasibility of the approaches, both simulations (control of tutorial system and position control of electropneumatic actuator model) are presented in Section 5.

\section{Preliminaries}

\subsection{Problem statement}

Consider the nonlinear uncertain system

$$
\dot{x}=f(x)+g(x) \cdot u
$$

with $x \in \mathcal{X} \subset \mathbb{R}^{n}$ the state vector and $u \in \mathbb{R}$ the control input. Functions $f(x)$ and $g(x)$ are smooth uncertain functions and are bounded for $x \in \mathcal{X}$; furthermore, $f(x)$ contains unmeasured perturbations term and $g(x) \neq 0$ for $x \in \mathcal{X}$ (i.e. system (1) is controllable for all $x \in \mathcal{X})$. The control objective consists in forcing the continuous function $\sigma(x, t)$, named 
sliding variable, to 0 . Supposing that $\sigma$ admits a relative degree equal to 1 with respect to $u$, one gets

$$
\begin{aligned}
\dot{\sigma} & =\frac{\partial \sigma}{\partial x} \dot{x}+\frac{\partial \sigma}{\partial t}=\underbrace{\frac{\partial \sigma}{\partial t}+\frac{\partial \sigma}{\partial x} \cdot f(x)}_{\Psi(x, t)}+\underbrace{\frac{\partial \sigma}{\partial x} \cdot g(x)}_{\Gamma(x, t)} \cdot u \\
& =\Psi(x, t)+\Gamma(x, t) \cdot u
\end{aligned}
$$

Functions $\Psi(x, t)$ and $\Gamma(x, t)$ are supposed to be such that

$$
|\Psi| \leq \Psi_{M}, 0<\Gamma_{m} \leq \Gamma \leq \Gamma_{M}
$$

for $x \in \mathcal{X}$. It is assumed that $\Psi_{M}, \Gamma_{m}$ and $\Gamma_{M}$ exist but are not known. The objective of the paper is to propose a new sliding mode controller $u(\sigma, t)$ with the same features as classical SMC (robustness, finite time convergence) but without any information on uncertainties and perturbations (appearing in $f(x)$ ); this latter is only known to be bounded. Furthermore, this objective allows to ensure a global stability of closed-loop system whereas the classical way (with knowledge of uncertainties bounds) only ensures its semi-global stability. In the sequel, definitions of ideal and real sliding mode are recalled.

Definition 1 [14] Consider the nonlinear system (1), and let the system be closed by some possibly-dynamical discontinuous feedback. Variable $\sigma$ is continuous function, and the set

$$
\mathcal{S}=\{x \in \mathcal{X} \mid \sigma(x, t)=0\},
$$

called "sliding surface", is non-empty and is locally an integral set in the Filippov sense [7], i.e. it consists of Filippov's trajectories of the discontinuous dynamical system. The motion on $\mathcal{S}$ is called "sliding mode" with respect to the sliding variable $\sigma$.

In real applications, an "ideal" sliding mode as defined in Definition 1 can not be established. Then, it is necessary to introduce the concept of "real" sliding mode.

Definition 2 [14] Given the sliding variable $\sigma(x, t)$, the "real sliding surface" associated to (1) is defined as (with $\delta>0$ )

$$
\mathcal{S}^{*}=\{x \in \mathcal{X}|| \sigma \mid<\delta\} .
$$

Definition 3 [14] Consider the non-empty real sliding surface $\mathcal{S}^{*}$ given by (4), and assume that it is locally an integral set in the Filippov sense. The corresponding behavior of system (1) on (4) is called "real sliding mode" with respect to the sliding variable $\sigma(x, t)$.

\subsection{Discussion of adaptive sliding mode control solutions and motivations}

Two adaptive sliding mode controllers have been given in [10] and [13] and are going now to be discussed. These two approaches follow two different ways : the first does not require the knowledge of uncertainties/perturbations bound and consists in increasing the control gain since a sliding mode is established whereas the second one requires the knowledge of the bound and is using the equivalent control concept in order to evaluate and to minimize the control gain. Then, a same objective can be claimed, which is the dynamical adaptation of the gain in order to counteract perturbations and uncertainties. 
Theorem 1 ([10]) Given the nonlinear uncertain system (1) with the sliding variable $\sigma(x, t)$ dynamics (2) controlled by

$$
u=-K(t) \cdot \operatorname{sign}(\sigma(x, t))
$$

with the adaptation law $K(t)$ given by

$$
\dot{K}=\bar{K} \cdot|\sigma(x, t)|
$$

with $\bar{K}>0$ and $K(0)>0$, then there exists a finite time $t_{F} \geq 0$ so that a sliding mode is established in system for all $t \geq t_{F}$, i.e. $\sigma(x, t)=0$ for $t \geq t_{F}$.

Discussion. The main feature of this approach is that it does not require a priori the knowledge of control gain. However, from $K$-dynamics, it yields that when $\sigma=0, \dot{K}=0$. In this case, the gain $K$ is clearly over-estimated with respect to uncertainties, which induces larger chattering (see simulations results in [10]-Section III). Furthermore, this methodology is applicable only for "ideal" sliding mode, the objective $\sigma=0$ being reachable. In case of real sliding mode, this latter objective is not reachable which induces that $K$ gain is always increasing. In [10], authors propose to modify $K$-dynamics by introducing a boundary layer neighboring the sliding surface $\sigma=0$. It means that accuracy has to be sacrificed in order to apply the previous controller and that the control gain is still over-estimated. Also, with discrete-time measurements, adaptation law (5) inevitably implies the tending of $K$ to infinity, if the sampling interval is separated from zero.

Theorem 2 ([13]) Given the nonlinear uncertain system (1) with the sliding variable $\sigma(x, t)$ dynamics (2) controlled by

$$
u=-K(t) \cdot \operatorname{sign}(\sigma(x, t))
$$

with the adaptation law $K(t)$ given by

$$
K(t)=\bar{K} \cdot|\eta|+\chi
$$

with $\bar{K}>0, \chi>0$ and $\eta$ the average of $\operatorname{sign}(\sigma)$ obtained through a low pass-filter

$$
\tau \cdot \dot{\eta}+\eta=\operatorname{sign}(\sigma(x, t))
$$

with $\tau>0$, if

$$
\bar{K} \geq\left|\frac{\Psi}{\Gamma}\right|,
$$

then there exists a finite time $t_{F}>0$ so that the sliding mode is established for all $t \geq t_{F}$.

Discussion. The main feature of this controller is the adjustment of the control gain by using the equivalent control concept. It implies that chattering is decreasing. However, $K$ adaptation law requires the knowledge of uncertainties/perturbations bounds. Furthermore, the use of low-pass filter introduces in the closed-loop system dynamics and transient phenomena in case of perturbations. Note also that methodologies for the tuning of parameters $\tau$ and $\chi$ have not been detailed except their positivity and the fact that the time constant of the low pass filter $\tau$ must be sufficiently small. However, their tuning could engender very different behaviors of closed-loop system.

Motivations. The main objective of the current paper consists in providing new adaptive sliding mode controllers such that 
- uncertainties/perturbation bounds exist but are not known.

- the gain adaptation law does not overestimate uncertainties/perturbations magnitude and then, the obtain control magnitude is reasonable.

A first solution to the problem under interest in this paper consists in combining the two previous gain adaptation laws: the first one would be used since a sliding mode is established, whereas the second allows to decrease the control gain once sliding mode is established. A second solution is an original adaptive real sliding mode control law that allows not to overestimate the gain value without using concept of equivalent control.

\section{Main results}

In this section, two adaptive sliding mode control algorithms that establish the sliding modes in SISO systems with bounded perturbations which bounds are unknown, are presented. The first algorithm combines the adaptive schemes formulated in Theorems 1-2 - Section 2, while mitigating the deficiencies of the combined gain-adaptation schemes. The second adaptive sliding mode control algorithm is completely original. It does not require the estimation of the perturbations via equivalent control as in [13] (Theorem 2) and does not overestimate the control gain as in [10] (Theorem 1). Furthermore, the second adaptive-gain sliding mode control algorithm requires smaller amount of tuning parameters than the first algorithm, and is developed in the real sliding mode context.

\subsection{First adaptive sliding mode control law}

Consider the following controller

$$
u=-K \cdot \operatorname{sign}(\sigma(x, t))
$$

with the gain $K(t)$ defined by ( $\epsilon$ being a positive parameter)

- If $|\sigma| \neq 0, K(t)$ is the solution of

$$
\dot{K}=\bar{K}_{1} \cdot|\sigma(x, t)|
$$

with $\bar{K}_{1}>0$ and $K(0)>0$.

- If $\sigma=0, K(t)$ reads as

$$
\begin{aligned}
& K(t)=\bar{K}_{2} \cdot|\eta|+\bar{K}_{3} \\
& \tau \dot{\eta}+\eta=\operatorname{sign}(\sigma(x, t))
\end{aligned}
$$

with $\bar{K}_{2}=K\left(t^{*}\right), \bar{K}_{3}>0$ and $\tau>0 . t^{*}$ is the largest time value such that, by denoting $t^{*-}$ the time just before $t^{*}, \sigma\left(x\left(t^{*-}\right), t^{*-}\right) \neq 0$ and $\sigma\left(x\left(t^{*}\right), t^{*}\right)=0$.

Discussion. By supposing that $|\sigma(x(0), 0)| \neq 0$, the adaptive sliding mode control law (7)-(9) works as follows

- The gain $K(t)$ is increasing due to the adaptation law (8) up to a value large enough to counteract the bounded uncertainty with unknown bounds in (1) until the sliding mode starts. Denote the time instant when the sliding mode starts for the first time as $t_{1}$. 
- As sliding mode has started, i.e. $\sigma(x(t), t)=0$, from $t=t_{1}, K(t)$ follows the gain adaptation law (9). Then, gain $K(t)$ is adapted through (9) with $\bar{K}_{2}=K\left(t_{1}\right)$. Note that this strategy will allow to decrease the gain and then to adjust it with respect to the current uncertainties/perturbations.

- However, if the varying uncertainty/perturbation exceeds the value $\bar{K}_{2}=K\left(t_{1}\right)$, then the sliding mode will be destroyed and $\sigma(x(t), t)$ becomes not equal to zero. Next, the gain adaptation will happen in accordance with (8). The gain $K(t)$ will be increasing until the sliding mode occurs again at the reaching time instant $t_{2}$.

- As sliding mode has occured and $\sigma(x(t), t)=0$ from $t=t_{2}, K(t)$ now follows the gain adaptation law (9) with $\bar{K}_{2}=K\left(t_{2}\right)$. And so on $\ldots{ }^{1}$

Lemma 1 Given the nonlinear uncertain system (1) with the sliding variable $\sigma(x, t)$ dynamics (2) controlled by (7),(8),(9), the gain $K(t)$ has an upper-bound, i.e. there exists a positive constant $K^{*}$ so that

$$
K(t) \leq K^{*}, \quad \forall t>0
$$

Proof of Lemma 1 is in Appendix section.

Theorem 3 Given the nonlinear uncertain system (1) with the sliding variable $\sigma(x, t) d y$ namics (2) controlled by (7),(8),(9), there exists a finite time $t_{F}>0$ so that a sliding mode is established for all $t \geq t_{F}$, i.e. $\sigma(x, t)=0$ for $t \geq t_{F}$.

Proof. The proof is composed by two steps. The first step concerns $\sigma \neq 0$ which yields $K$-dynamics described by (8), whereas the second one concerns $\sigma=0$ with $K$-dynamics described by (9).

- Suppose that $\sigma(0) \neq 0$ : in this case, $K(t)$-dynamics read as (8). Consider the following Lyapunov candidate function, with $K^{*}$ a positive constant

$$
V=\frac{1}{2} \sigma^{2}+\frac{1}{2 \gamma}\left(K-K^{*}\right)^{2}
$$

One has

$$
\begin{aligned}
\dot{V} & =\sigma \cdot \Psi-\sigma \cdot \Gamma \cdot K \cdot \operatorname{sign}(\sigma)+\frac{1}{\gamma}\left(K-K^{*}\right) \cdot \bar{K}_{1} \cdot|\sigma| \\
& \leq \Psi_{M} \cdot|\sigma|-\Gamma_{m} \cdot K \cdot|\sigma|+\frac{1}{\gamma}\left(K-K^{*}\right) \cdot \bar{K}_{1} \cdot|\sigma| \\
& =\Psi_{M} \cdot|\sigma|-\Gamma_{m} \cdot K \cdot|\sigma|+\Gamma_{m} \cdot K^{*} \cdot|\sigma|-\Gamma_{m} \cdot K^{*} \cdot|\sigma|+\frac{1}{\gamma}\left(K-K^{*}\right) \cdot \bar{K}_{1} \cdot|\sigma| \\
& =\left(\Psi_{M}-\Gamma_{m} \cdot K^{*}\right) \cdot|\sigma|+\left(K-K^{*}\right) \cdot\left(-\Gamma_{m} \cdot|\sigma|+\frac{\bar{K}}{\gamma} \cdot|\sigma|\right)
\end{aligned}
$$

\footnotetext{
${ }^{1}$ It yields that $t^{*}$ defined just after $(9)$ equals first-of-all $t_{1}$, then $t_{2}$. Furthermore, in implementation case, $t^{*}$ is on-line determined by comparing, at each time $t, \sigma\left(x\left(t^{-}\right), t^{-}\right)$and $\sigma(x(t), t)=0$.
} 
From Lemma 1, there always exists $K^{*}>0$ such that $K(t)-K^{*}<0$ for all $t>0$. It yields

$$
\dot{V}=-\underbrace{\left(\Gamma_{m} \cdot K^{*}-\Psi_{M}\right)}_{\beta_{\sigma}} \cdot|\sigma|-\underbrace{\left(-\Gamma_{m} \cdot|\sigma|+\frac{\bar{K}}{\gamma} \cdot|\sigma|\right)}_{\beta_{K}} \cdot\left|K-K^{*}\right|
$$

There always exists $K^{*}$ and $\gamma$ such that $K^{*}>\frac{\Psi_{M}}{\Gamma_{m}}$ and $\gamma<\frac{\bar{K}}{\Gamma_{m}}$, which yields $\beta_{\sigma}>0$ and $\beta_{K}>0$. Then, one gets

$$
\begin{aligned}
\dot{V} & =-\beta_{\sigma} \cdot \sqrt{2} \frac{|\sigma|}{\sqrt{2}}-\beta_{K} \cdot \sqrt{2 \gamma} \frac{\left|K-K^{*}\right|}{\sqrt{2 \gamma}} \\
& \leq-\min \left\{\beta_{\sigma} \sqrt{2}, \beta_{K} \sqrt{2 \gamma}\right\}\left(\frac{|\sigma|}{\sqrt{2}}+\frac{\left|K-K^{*}\right|}{\sqrt{2 \gamma}}\right) \leq-\beta \cdot V^{1 / 2}
\end{aligned}
$$

with $\beta=\sqrt{2} \min \left\{\beta_{\sigma}, \beta_{K} \sqrt{\gamma}\right\}$. Therefore, finite time convergence to a domain $\sigma=0$ is guaranteed from any initial condition $|\sigma(0)|>0$, and the reaching time $t_{r}$ can be easily estimated as

$$
t_{r} \leq \frac{2 V(0)^{1 / 2}}{\beta} .
$$

- Suppose now that $\sigma(0)=0$ : Theorem 1 of [13] claims that, if $K$ is large enough with respect to uncertainties/perturbations effects, then sliding mode control (7) with the gain adaptation algorithm (9) allows keeping trajectories of system (1) on the sliding surface $\sigma=0$. Given that trajectories of system (1) reach $\sigma=0$, it means that $K$ is large enough as required by Theorem 1 of [13]. Then, the sliding mode is established in system (1) for all $t \geq t_{r}$. Theorem 3 is proven.

Implementation issues. The proposed adaptive-gain sliding mode control algorithm in (7)-(8)-(9) is not ready for the practical implementations. In fact, it is not possible to reach the objective $\sigma=0$ due to sampled computation, noisy measurements or other non-idealities. That is why it is important considering the implementation of the previous controller in a real sliding mode context. The following modifications of the gain-adaptation algorithm (7)-(8)-(9) is proposed $^{2}$

- If $|\sigma(x, t)|>\epsilon>0, K(t)$ is the solution of

$$
\dot{K}=\bar{K}_{1} \cdot|\sigma(x, t)|
$$

with $\bar{K}_{1}>0$ and $K(0)>0$.

- If $|\sigma(x, t)| \leq \epsilon, K(t)$ reads as

$$
\begin{aligned}
& K(t)=\bar{K}_{2} \cdot|\eta|+\bar{K}_{3} \\
& \tau \dot{\eta}+\eta=\operatorname{sign}(\sigma(x, t))
\end{aligned}
$$

with $\bar{K}_{2}=K\left(t^{*}\right), \bar{K}_{3}>0$ and $\tau>0 . t^{*}$ is the largest time value such that, by denoting $t^{*-}$ the time just before $t^{*},\left|\sigma\left(x\left(t^{*-}\right), t^{*-}\right)\right|>\epsilon$ and $\left|\sigma\left(x\left(t^{*}\right), t^{*}\right)\right| \leq \epsilon$.

\footnotetext{
${ }^{2}$ The parameter $\epsilon$ can be time-varying, as in the next section. Its tuning is detailed in Section 4 .
} 
Corollary 1 Given the nonlinear uncertain system (1) with the sliding variable $\sigma(x, t)) d y$ namics (2) controlled by (7),(13),(14), there exists a finite time $t_{F}>0$ so that a real sliding mode is established for all $t \geq t_{F}$, i.e. $|\sigma(x, t)|<\epsilon$ for $t \geq t_{F}$.

Proof. The proof is composed by two steps. The first step concerns $|\sigma|>\epsilon$ which yields $K$-dynamics described by (13), whereas the second one concerns $|\sigma| \leq \epsilon$ with $K$-dynamics described by (14). Then, the proof follows the same way than the proof of Theorem 3 based on Lyapunov analysis when $|\sigma|>\epsilon$, and on Theorem 1 of [13] when $|\sigma| \leq \epsilon$.

\subsection{Second adaptive sliding mode control law}

The first adaptive sliding mode control law uses concept of equivalent control which introduces low-pass filter dynamics with $\tau$ parameter that is not easy to tune [25]. The controller displayed in this section does not estimate the boundary of perturbation and uncertainties. But, there is an eminent price to do that: the new strategy guarantees a real sliding mode only. Consider the following controller

$$
u=-K \cdot \operatorname{sign}(\sigma(x, t))
$$

with the gain $K(t)$ defined such that

$$
\dot{K}= \begin{cases}\bar{K} \cdot|\sigma(x, t)| \cdot \operatorname{sign}(|\sigma(x, t)|-\epsilon) & \text { if } K>\mu \\ \mu & \text { if } K \leq \mu\end{cases}
$$

with $K(0)>0, \bar{K}>0, \epsilon>0$ and $\mu>0$ very small. The parameter $\mu$ is introduced in order to get only positive values for $K$. In the sequel, for discussion and proof, and without loss of generality but for a sake of clarity, one supposes that $K(t)>\mu$ for all $t>0$.

Discussion. Once sliding mode with respect to $\sigma(x, t)$ established, the proposed gain adaptation law (16) allows the gain $K$ declining (while $|\sigma(x, t)|<\epsilon$ ). In the other words, the gain $K$ will be kept at the smallest level that allows a given accuracy of $\sigma$-stabilization. Of course, as described in the sequel, this adaptation law allows to get an adequate gain with respect to uncertainties/perturbations magnitude.

Lemma 2 Given the nonlinear uncertain system (1) with the sliding variable $\sigma(x, t)$ dynamics (2) controlled by (15),(16), the gain $K(t)$ has an upper-bound, i.e. there exists a positive constant $K^{*}$ so that

$$
K(t) \leq K^{*}, \quad \forall t>0 .
$$

Proof of Lemma 2 is in Appendix section.

Theorem 4 Given the nonlinear uncertain system (1) with the sliding variable $\sigma(x, t) d y$ namics (2) controlled by (15),(16), there exists a finite time $t_{F}>0$ so that a real sliding mode is established for all $t \geq t_{F}$, i.e. $|\sigma(x, t)|<\delta$ for $t \geq t_{F}$, with

$$
\delta=\sqrt{\epsilon^{2}+\frac{\Psi_{M}^{2}}{\bar{K} \Gamma_{m}}} .
$$


Proof. The proof is based on Lyapunov's approach and shows that, when $|\sigma|>\epsilon$, then control strategy ensures that $|\sigma|<\epsilon$ in a finite time. Furthermore, it is proven that as soon as $\sigma$ reaches the domain $|\sigma| \leq \epsilon$, it stays in the domain $|\sigma| \leq \delta$ defined by (17) for all consecutive time. Therefore, the proof shows that the real sliding mode is established in finite time in the domain $|\sigma| \leq \delta$.

Consider the following Lyapunov candidate function

$$
V=\frac{1}{2} \sigma^{2}+\frac{1}{2 \gamma}\left(K-K^{*}\right)^{2}
$$

One has

$$
\begin{aligned}
\dot{V} & =\sigma \cdot \Psi-\sigma \cdot \Gamma \cdot K \cdot \operatorname{sign}(\sigma)+\frac{1}{\gamma}\left(K-K^{*}\right) \cdot \bar{K} \cdot|\sigma| \cdot \operatorname{sign}(|\sigma|-\epsilon) \\
& \leq \Psi_{M} \cdot|\sigma|-\Gamma_{m} \cdot K \cdot|\sigma|+\frac{1}{\gamma}\left(K-K^{*}\right) \cdot \bar{K} \cdot|\sigma| \cdot \operatorname{sign}(|\sigma|-\epsilon) \\
& =\Psi_{M} \cdot|\sigma|-\Gamma_{m} \cdot K \cdot|\sigma|+\Gamma_{m} \cdot K^{*} \cdot|\sigma|-\Gamma_{m} \cdot K^{*} \cdot|\sigma|+\frac{1}{\gamma}\left(K-K^{*}\right) \cdot \bar{K} \cdot|\sigma| \cdot \operatorname{sign}(|\sigma|-\epsilon) \\
& =\left(\Psi_{M}-\Gamma_{m} \cdot K^{*}\right) \cdot|\sigma|+\left(K-K^{*}\right) \cdot\left(-\Gamma_{m} \cdot|\sigma|+\frac{\bar{K}}{\gamma} \cdot|\sigma| \cdot \operatorname{sign}(|\sigma|-\epsilon)\right)
\end{aligned}
$$

Introduce parameter $\beta_{K}>0$ as

$$
\begin{aligned}
\dot{V}= & \left(\Psi_{M}-\Gamma_{m} \cdot K^{*}\right) \cdot|\sigma|+\left(K-K^{*}\right) \cdot\left(-\Gamma_{m} \cdot|\sigma|+\frac{\bar{K}}{\gamma} \cdot|\sigma| \cdot \operatorname{sign}(|\sigma|-\epsilon)\right) \\
& +\beta_{K} \cdot\left|K-K^{*}\right|-\beta_{K} \cdot\left|K-K^{*}\right|
\end{aligned}
$$

From Lemma 2, there always exists $K^{*}>0$ such that $K(t)-K^{*}<0$ for all $t>0$. It yields

$$
\begin{aligned}
\dot{V}= & -\underbrace{\left(-\Psi_{M}+\Gamma_{m} \cdot K^{*}\right)}_{\beta_{\sigma}>0} \cdot|\sigma|-\beta_{K} \cdot\left|K-K^{*}\right| \\
& -\underbrace{\left(-\Gamma_{m} \cdot|\sigma|+\frac{\bar{K}}{\gamma} \cdot|\sigma| \cdot \operatorname{sign}(|\sigma|-\epsilon)-\beta_{K}\right) \cdot\left|K-K^{*}\right|}_{\xi}
\end{aligned}
$$

Then, one gets

$$
\begin{aligned}
\dot{V} & =-\beta_{\sigma} \cdot|\sigma|-\beta_{K} \cdot\left|K-K^{*}\right|-\xi \\
& =-\beta_{\sigma} \cdot \sqrt{2} \frac{|\sigma|}{\sqrt{2}}-\beta_{K} \cdot \sqrt{2 \gamma} \frac{\left|K-K^{*}\right|}{\sqrt{2 \gamma}}-\xi \\
& \leq-\min \left\{\beta_{\sigma} \sqrt{2}, \beta_{K} \sqrt{2 \gamma}\right\}\left(\frac{|\sigma|}{\sqrt{2}}+\frac{\left|K-K^{*}\right|}{\sqrt{2 \gamma}}\right)-\xi \leq-\beta \cdot V^{1 / 2}-\xi
\end{aligned}
$$

with $\beta=\sqrt{2} \min \left\{\beta_{\sigma}, \beta_{K} \sqrt{\gamma}\right\}$.

- Case 1. Suppose that $|\sigma|>\epsilon$. $\xi$ is positive if

$$
-\Gamma_{m} \cdot|\sigma|+\frac{\bar{K}}{\gamma} \cdot|\sigma|-\beta_{K}>0 \Rightarrow \gamma<\frac{\bar{K} \cdot \epsilon}{\Gamma_{m} \cdot \epsilon+\beta_{K}}
$$


From (21), one gets

$$
\dot{V} \leq-\beta \cdot V^{1 / 2}-\xi \leq-\beta \cdot V^{1 / 2}
$$

It is always possible to choose $\gamma$ such that the previous inequality fulfills. Therefore, finite time convergence to a domain $|\sigma| \leq \epsilon$ is guaranteed from any initial condition $|\sigma(0)|>\epsilon$.

- Case 2. Suppose now that $|\sigma|<\epsilon$. Function $\xi$ in (20) can be negative. It means that $\dot{V}$ would be sign indefinite, and it is not possible to conclude on the closed-loop system stability. Therefore, $|\sigma|$ can increase over $\epsilon$. As soon as $|\sigma|$ becomes greater than $\epsilon, \dot{V} \leq$ $-\beta \cdot V^{1 / 2}$ and $V$ starts decreasing. Apparently, decrease of $V$ can be achieved via increase of $K$ allowing $|\sigma|$ to increase before it starts decreasing down to $|\sigma| \leq \epsilon$. Without loss of generality, let estimate the overshoot when $\sigma_{0}=\sigma(0)=\epsilon^{+}$and $K_{0}=\mathcal{K}(0)=K(0)>0$ : considering the "worst" case (with respect to uncertainties/perturbations), one has

$$
\begin{aligned}
\dot{\sigma} & =\Psi_{M}-K \cdot \Gamma_{m} \\
\dot{K} & =\bar{K} \cdot|\sigma|
\end{aligned}
$$

Then, it yields

$$
\begin{aligned}
\sigma(t) & =\sigma_{0} \cos \left(\sqrt{\bar{K} \Gamma_{m}} t\right)+\frac{\Psi_{M}-K_{0} \cdot \Gamma_{m}}{\sqrt{\bar{K} \Gamma_{m}}} \cdot \sin \left(\sqrt{\bar{K} \Gamma_{m}} t\right) \\
K(t) & =\sigma_{0} \sqrt{\frac{\bar{K}}{\Gamma_{m}}} \sin \left(\sqrt{\bar{K} \Gamma_{m}} t\right)+\left(K_{0}-\frac{\Psi_{M}}{\Gamma_{m}}\right) \cos \left(\sqrt{\bar{K} \Gamma_{m}} t\right)+\frac{\Psi_{M}}{\Gamma_{m}}
\end{aligned}
$$

Then, one gets

$$
\begin{aligned}
\sigma(t) & =\sqrt{\sigma_{0}^{2}+\frac{\left(\Psi_{M}-K_{0} \cdot \Gamma_{m}\right)^{2}}{\bar{K} \Gamma_{m}}} \sin \left(\sqrt{\bar{K} \Gamma_{m}} t+\Theta_{\sigma}\right) \\
K(t) & =\sqrt{\sigma_{0}^{2} \frac{\bar{K}}{\Gamma_{m}}+\left(K_{0}-\frac{\Psi_{M}}{\Gamma_{m}}\right)^{2}} \sin \left(\sqrt{\bar{K} \Gamma_{m}} t+\Theta_{K}\right)+\frac{\Psi_{M}}{\Gamma_{m}}
\end{aligned}
$$

It appears from (26) that, when $\sigma_{0}=\epsilon^{+} \rightarrow \epsilon$, the maximum value $\delta$ of $\sigma_{M}$ reads as

$$
\delta=\sqrt{\epsilon^{2}+\frac{\Psi_{M}^{2}}{\bar{K} \Gamma_{m}}}
$$

In conclusion, $\sigma$ converges to the domain $|\sigma| \leq \epsilon$ in a finite time, but could be sustained in the bigger domain $|\sigma| \leq \delta$. Therefore, the real sliding mode exists in the domain $|\sigma| \leq \delta$.

\section{On $\epsilon$-tuning}

The objective of this section consists in providing a methodology for the tuning of parameter $\epsilon$ for the both algorithms in case of sampled controllers. In fact, the choice of parameter $\epsilon$ has to be made by an adequate way because a "bad" tuning could provide either unstability and control gain increasing to infinity, or bad accuracy for closed-loop system as described in the sequel. 
- If parameter $\epsilon$ is too small, and due to large gain $K$ and sampling period $T_{e}$, system trajectories are such that $|\sigma|$ never stays lower than $\epsilon$. From $K$-dynamics (13) or (16), it yields that gain $K$ is increasing, which induces larger oscillation, and so on ...

- If parameter $\epsilon$ is too large, system trajectories are such that, in spite of large gain $K$ and sampling period $T_{e},|\sigma|$ is evolving around $\epsilon$, it follows that controller accuracy is not as good as possible.

Note that $\epsilon$ should rather be too large than too small because, in the first case, closed-loop system trajectories are globally uniformly ultimately bounded even if accuracy is bad.

Parameter $\epsilon$ has to be tuned such that, as long as $K(t)$ is greater than $|\Psi / \Gamma|,|\sigma|<\epsilon$. In this case, given that gain $K(t)$ is sufficient to counteract the perturbations, there is no reason that $\sigma$ increases over $\epsilon$. Suppose that, at $t=t_{1},\left|\sigma\left(t_{1}\right)\right| \leq \epsilon$ and

$$
K\left(t_{1}\right) \geq\left|\frac{\Psi\left(t_{1}\right)}{\Gamma\left(t_{1}\right)}\right| .
$$

It is clear that control gain $K$ is sufficient to ensure

$$
\left|\sigma\left(t_{1}+T_{e}\right)\right| \leq \epsilon
$$

From Euler's formula, one has

$$
\sigma\left(t_{1}+T_{e}\right) \sim \sigma\left(t_{1}\right)+\left[\Psi\left(t_{1}\right)-\Gamma\left(t_{1}\right) \cdot K\left(t_{1}\right) \cdot \operatorname{sign}\left(\sigma\left(t_{1}\right)\right] \cdot T_{e}\right.
$$

From (28), one has

$$
|\Psi| \leq|\Gamma| \cdot K
$$

It yields

$$
\left|\Psi\left(t_{1}\right)-\Gamma\left(t_{1}\right) \cdot K\left(t_{1}\right) \cdot \operatorname{sign}\left(\sigma\left(t_{1}\right)\right)\right| \leq 2 \Gamma_{M} \cdot K\left(t_{1}\right) .
$$

Furthermore, as gain $K(t)$ fulfills (28), $\dot{\sigma}$-sign is the opposite of $\sigma$ sign. It means that, if $0 \leq \sigma\left(t_{1}\right) \leq \epsilon$ (resp. $\left.-\epsilon \leq \sigma \leq 0\right)$, function $\sigma$ will decrease (resp. increase). Then, in order to guarantee that $\left|\sigma\left(t_{1}+T_{e}\right)\right|$ will not exceed $\epsilon$, the "worst" case is $\sigma\left(t_{1}\right)=0$. From (29), it yields

$$
\left|\sigma\left(t_{1}+T_{e}\right)\right| \leq 2 \Gamma_{M} \cdot K\left(t_{1}\right) T_{e}
$$

Given that it must be ensured $\left|\sigma\left(t_{1}+T_{e}\right)\right| \leq \epsilon$, and as the best accuracy and the closed-loop system are the both objectives, $\epsilon$ has to be chosen as a time-varying function equal to the smallest admissible value

$$
\epsilon(t)=2 \Gamma_{M} \cdot K\left(t_{1}\right) T_{e} .
$$

Discussion. Equation (31) means that the knowledge of bounds of $\Gamma$ is required for the choice of $\epsilon$ in case of sampling controller. However, note that this latter equation gives the best tuning. As said previously, $\epsilon$ can be tuned to a "too" large value which guarantees the stability but with a worst accuracy. Furthermore, the equation (31) gives at least a methodology for $\epsilon$-computation: no such information is given in [10] whereas a such parameter is required for the boundary layer in case of practical implementation of the controller.

However, in case of many practical applications, the knowledge of $\Gamma$ bounds is not really required. In fact, when applied, the controller design procedure is very often the following: 
uncertain functions read as $\Psi=\Psi_{N o m}+\Delta \Psi$ and $\Gamma=\Gamma_{N o m}+\Delta \Gamma$ where $\Psi_{N o m}$ and $\Gamma_{N o m}$ are the nominal known functions and $\Delta \Psi$ and $\Delta \Gamma$ their unknown parts. Furthermore, in most of the cases, one has $\left|\Psi_{N o m}\right| \geq|\Delta \Psi|$ and $\left|\Gamma_{N o m}\right| \geq|\Delta \Gamma|$. Then, by supposing that $\Psi_{N o m}$ is invertible, the control law ${ }^{3}$

$$
u=\frac{-\Psi_{N o m}+v}{\Gamma_{N o m}}
$$

is applied to system (2) which gives

$$
\dot{\sigma}=\left(\Delta \Psi+\frac{\Psi_{N o m}}{\Gamma_{N o m}} \Delta \Gamma\right)+\left(1-\frac{\Delta \Gamma}{\Gamma_{N o m}}\right) \cdot v
$$

As $\left|\Gamma_{N o m}\right| \geq|\Delta \Gamma|$, one gets $\left|1-\frac{\Delta \Gamma}{\Gamma_{N o m}}\right| \leq 2$. From (31), it yields

$$
\epsilon(t)=4 K(t) T_{e}
$$

\section{Simulations}

\subsection{Tutorial}

Consider the following uncertain system

$$
\dot{\sigma}=\Psi(t)+u
$$

Function $\Psi(t)$ is an uncertain bounded function described by Figure 1. Simulations have

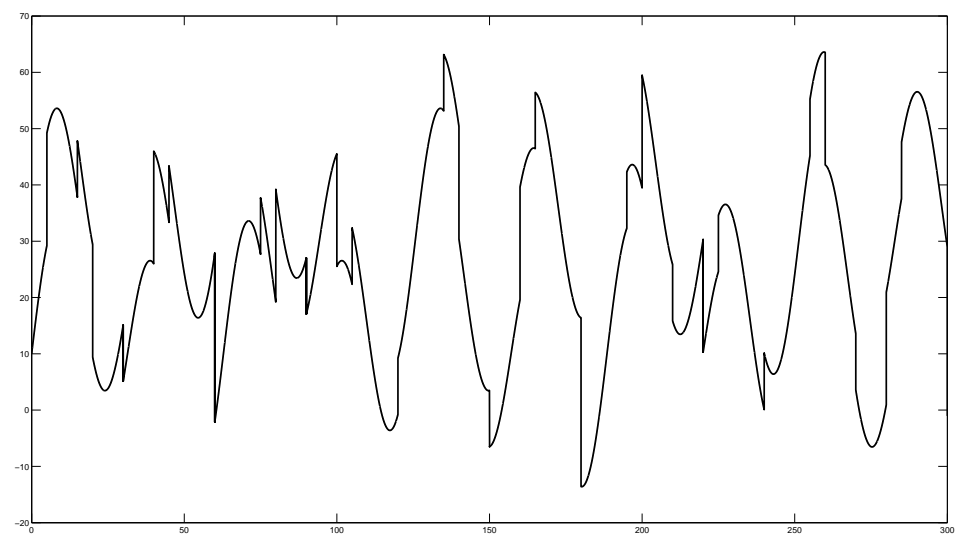

Figure 1: Unknown term $\Psi$ versus time (sec).

been made by supposing that $\sigma(0)=10$ and $T_{e}=0.0001 \mathrm{sec}$. From (31), parameter $\epsilon$ reads as $\epsilon(t)=2 K(t) \cdot T_{e}$.

\footnotetext{
${ }^{3}$ In [6], it has been shown that, for such class of uncertainties, this control law allows to decrease the influence of perturbations and uncertainties, and then to reduce the control gain.
} 
Control algorithm 1 in eqs. (7)-(13)-(14). Control input $u$ and gain adaptation law $K(t)$ respectively read as in eqs. (7)-(13)-(14). Gain is initialized at $K(0)=10$, its dynamics being tuned with $\bar{K}_{1}=1000, \bar{K}_{3}=1$ and $\tau=0.1$ sec.

Control algorithm 2 in eqs. (15)-(16). Control input $u$ and gain adaptation law $K(t)$ respectively read as in eqs. (15)-(16). Gain is initialized at $K(0)=10$, its dynamics being tuned with $\bar{K}=1000$ and $\mu=0.1$.
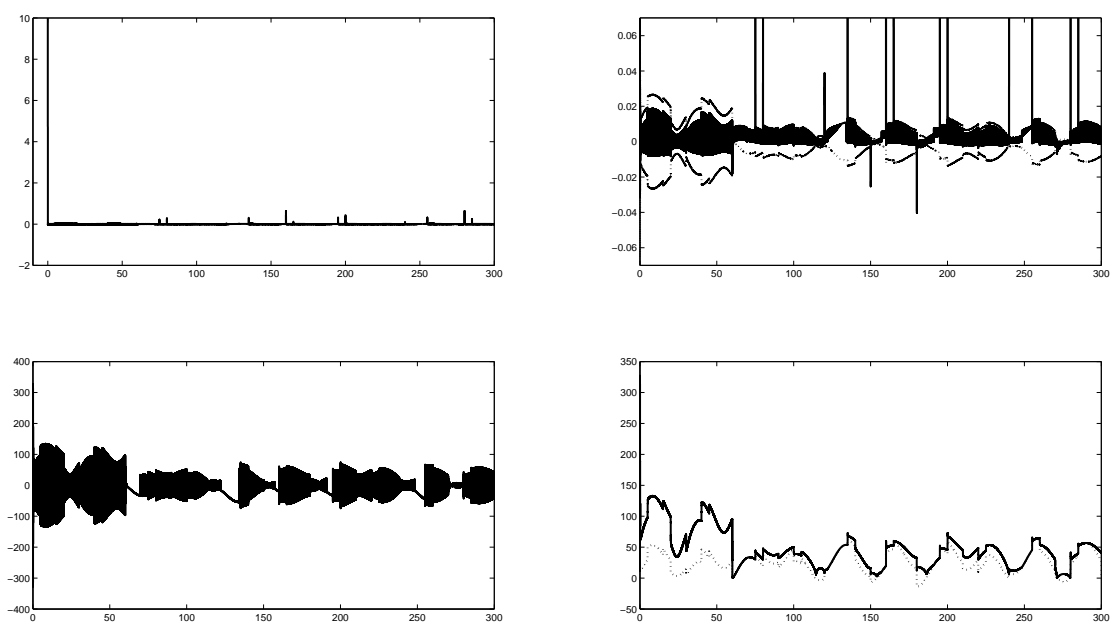

Figure 2: Control algorithm 1(7)-(13)-(14). Top-Left. Sliding variable $\sigma$ versus time (sec). Top-Right. Zoom on sliding variable $\sigma$ (solid line), $\epsilon(t)$ and $-\epsilon(t)$ (dotted lines) versus time (sec). Bottom-Left. Control input $u$ versus time (sec). Bottom-Right. Gain $K(t)$ (solid line) and perturbation $\Psi(t)$ (dotted line) versus time (sec).

Figures 2 and 3 display simulations results of both previous controllers applied to system (33). It appears that both control laws yield to very similar results. Based on simulation plots, one can conclude that both adaptation algorithmes provide the controller gain $K(t)$ to follow closely the perturbation $\Psi(t)$ which profile and boudary are not known a priori (see bottom-right plots in Figures 2 and 3). Therefore, the control gain $K(t)$ is not over-estimated and control chattering is minimal. Also, the sliding variable $\sigma$ is robustly constrainted to $|\sigma|<\epsilon(t)$ with exception of sparce spikes.

\subsection{Electropneumatic actuator}

The electropneumatic system under interest is a double acting actuator (Figure 4) composed by two chambers, denoted $P$ (as positive) and $N$ (as negative). The air mass flow rates entering the two chambers are modulated by two three-way servodistributors controlled with two electrical inputs of opposite signs $(u$ and $-u)$. The pneumatic jack horizontally moves a load carriage of mass $\mathrm{M}$ 

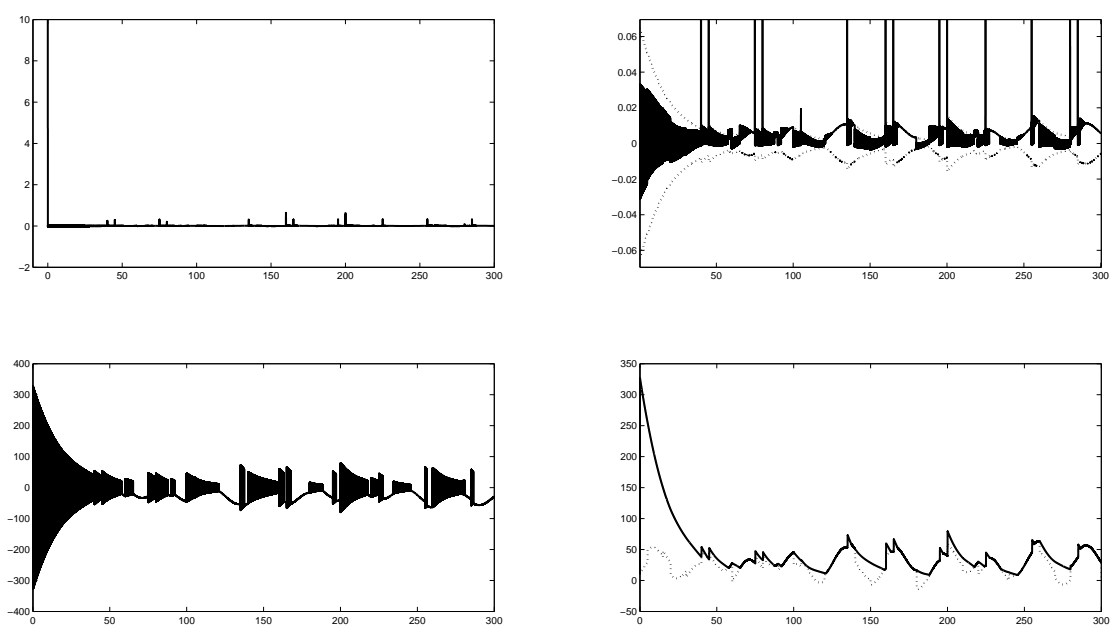

Figure 3: Control algorithm 2 (15)-(16). Top-Left. Sliding variable $\sigma$ versus time (sec). Top-Right. Zoom on sliding variable $\sigma$ (solid line), $\epsilon(t)$ and $-\epsilon(t)$ (dotted lines) versus time (sec). Bottom-Left. Control input $u$ versus time (sec). Bottom-Right. Gain $K(t)$ (solid line) and perturbation $\Psi(t)$ (dotted line) versus time (sec).

\subsubsection{Model}

Following standard assumptions on the pneumatic part of the electropneumatic system [21, $17,5,8,11]$, one gets a nonlinear dynamic model for the whole system

$$
\begin{aligned}
\dot{p}_{P} & =\frac{k r T}{V_{P}(y)}\left[q_{m N}\left(u, p_{p}\right)-\frac{S_{P}}{r T} p_{P} v\right] \\
\dot{p}_{N} & =\frac{k r T}{V_{N}(y)}\left[q_{m P}\left(-u, p_{N}\right)+\frac{S_{N}}{r T} p_{N} v\right] \\
\dot{v} & =\frac{1}{M}\left[S_{P} p_{P}-S_{N} p_{N}-b v-F_{f}-F_{e x t}\right] \\
\dot{y} & =v
\end{aligned}
$$

with $y$ the load carriage position, $v$ its velocity and $p_{P}$ and $p_{N}$ the pressures of $P$ and $N$ chambers. $V_{X}(X=P$ or $N)$ is the volume in the chamber $X, q_{m X}\left(u_{X}, p_{X}\right)$ the mass flow rate provided from the servodistributor $X, k$ the polytropic constant, $r$ the perfect gaz constant, $T$ the supply temperature and $S_{X}$ the piston area in the chamber $X$. The term $F_{f}$ represents all the dry friction forces which act on the moving part in presence of viscous friction $(b . v)$ and an external force only due to atmospheric pressure $\left(F_{\text {ext }}\right)$.

The model of mass flow rate delivered by each servodistributor can be reduced to a static function described by two relationships $q_{m P}\left(u, p_{P}\right)$ and $q_{m N}\left(-u, p_{N}\right)$. The two first equations of (34) concern the pneumatic part of the system and are obtained from the state equation of perfect gases, the mass conservation law and the polytropic law under the assumptions given above. The two last equations describe the mechanical part and are derived from 


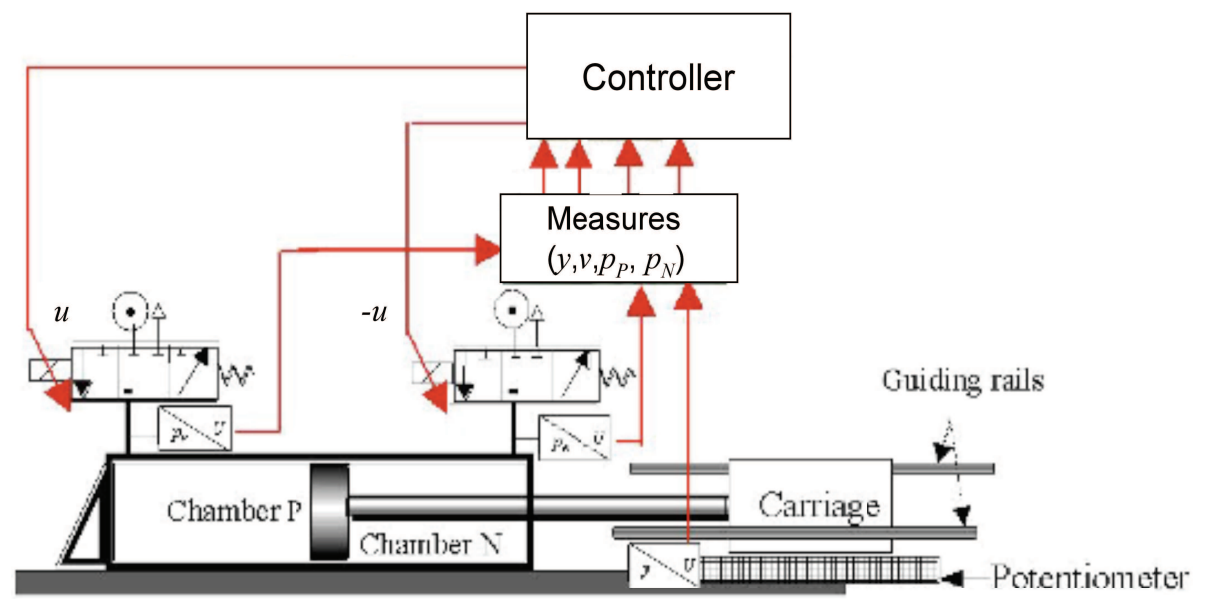

Figure 4: Electropneumatic system

the fundamental mechanical equation applied to the moving part. In order to get an affine nonlinear state model, the mass flow rate static characteristic issued from measurements [20] reads as $[2]$

$$
\begin{aligned}
& q_{m P}\left(u, p_{P}\right)=\varphi_{P}\left(p_{P}\right)+\psi_{P}\left(p_{P}, \operatorname{sgn}(u)\right) \cdot u \\
& q_{m N}\left(-u, p_{N}\right)=\varphi_{N}\left(p_{N}\right)-\psi_{N}\left(p_{N}, \operatorname{sgn}(-u)\right) \cdot u
\end{aligned}
$$

with functions $\varphi_{P}$ and $\psi_{P}$ (resp. $\psi_{N}$ and $\varphi_{N}$ ) $5^{\text {th }}$-order polynomial functions with respect to

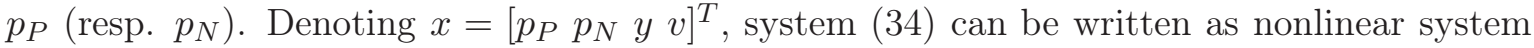
(1). Let define $\mathcal{X}$ as the physical domain

$\mathcal{X}=\left\{x \mid 1\right.$ bar $\leq p_{P} \leq 7$ bar, 1 bar $\leq p_{N} \leq 7$ bar, $-200 \mathrm{~mm} \leq y \leq 200 \mathrm{~mm},|v| \leq 1{\left.\mathrm{~m} . \mathrm{sec}^{-1}\right\}}$

It yields that, for $x \in \mathcal{X}$, system dynamics are bounded under a bounded control input $u$.

Two kinds of uncertainties are taken into account: uncertainties due to the identification of physical parameters, and perturbations. Viscous and dry friction coefficients have been identified, but the determination of their variations around their nominal values is a hard task. For example,the dry friction coefficient is difficult to identify because depending on the track surface quality (thus the piston position), the seal wear, the working conditions (temperature, pressure, quality of air) .... The mass flow rate delivered by each servodistributor has been approximated by polynomial functions (35); the uncertainties on $\varphi(\cdot)$ and $\psi(\cdot)$ have been evaluated to $\pm 15 \%$ and $\pm 5 \%$ respectively. Finally, during the load moving, the total mass in displacement can evolve from $17 \mathrm{~kg}$ until $47 \mathrm{~kg}$.

\subsubsection{Control design and simulations}

The aim of the control law is to get a good accuracy in term of position tracking for the desired trajectory defined in Figure 5. Following the previous section, consider the sliding variable

$$
\sigma=\lambda^{2} \cdot\left(y-y_{d}(t)\right)+2 \lambda \cdot\left(\dot{y}-\dot{y}_{d}(t)\right)+\left(\ddot{y}-\ddot{y}_{d}(t)\right)
$$




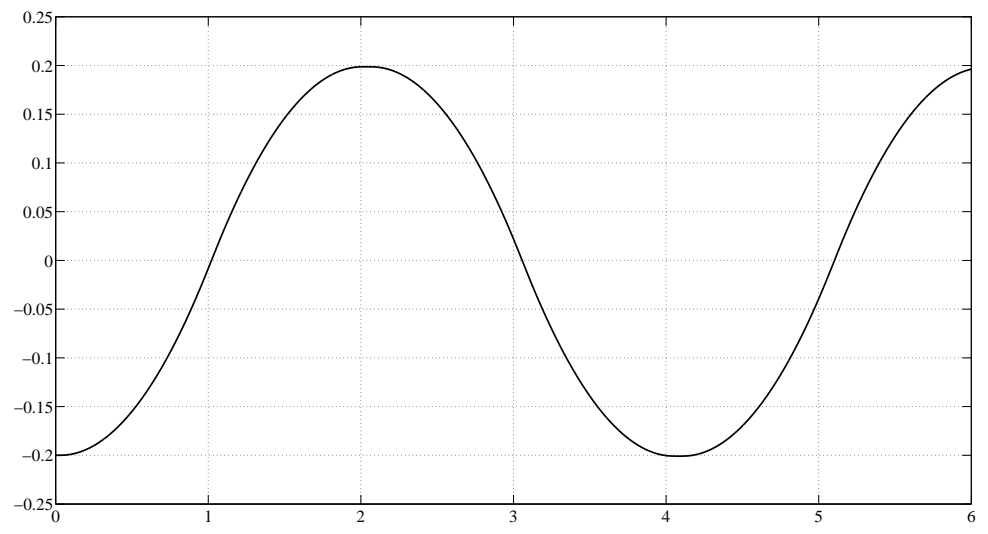

Figure 5: Desired position trajectory $y_{d}(t)(m)$ versus time $(s)$.

with $\lambda>0$. As system (34)-(35) admits a relative degree equal to 1 for $\sigma$ with respect to $u$, one gets ${ }^{4}$

$$
\dot{\sigma}=\Psi(\cdot)+\Gamma(\cdot) \cdot u
$$

As described in $[11,8]$, functions $\Psi(\cdot)$ and $\Gamma(\cdot)$ can be written as the sum of a "nominal" part $\left(\Psi_{N o m}\right.$ and $\left.\Gamma_{N o m}\right)$ and an "uncertain" one $(\Delta \Psi$ and $\Delta \Gamma)$, i.e.

$$
\Psi(\cdot)=\Psi_{N o m}(\cdot)+\Delta \Psi(\cdot), \quad \Gamma(\cdot)=\Gamma_{N o m}(\cdot)+\Delta \Gamma(\cdot),
$$

From [11, 8], functions $\Psi$ and $\Gamma$ are bounded for all $x \in \mathcal{X}$; furthermore, for all $x \in \mathcal{X}$, $\Gamma_{N o m}>0$. Then, $\Psi$ and $\Gamma$ fulfill (3). It yields that the control law reads as ${ }^{5}$

$$
u=\frac{1}{\Gamma_{N o m}}\left(-\Psi_{N o m}+v\right)
$$

with control input $v$ reading as previous control algorithms. Parameter $\epsilon$ reads as $\epsilon(t)=$ $4 K(t) \cdot T_{e}$. Parameters have been tuned as

$$
T_{e}=10^{-3} \text { sec, } \bar{K}=250, \bar{K}_{1}=50, K(0)=1, \lambda=33 .
$$

Furthermore, for Algorithm 1, one has

$$
\bar{K}_{3}=1, \tau=0.1 \mathrm{sec} .
$$

For Algorithm 2, one has $\mu=0.1$. Following simulations have been made by making variations of load mass $(+20 \%)^{6}$ and uncertainties on $\varphi$ and $\psi(-20 \%)$. Figures 6-7 display, for the both control laws, the actuator position with respect to desired trajectory (Top), the control input $u$ (Center), and the gain $K$ (Bottom). It appears that the both strategies yield to quite similar results. Note that, with the proposed gain tuning, Algorithm 1 induces lower magnitudes of control and gain in this time interval; in fact, Algorithm 2 gain is increasing to a larger value and takes time to decrease to similar values of Algorithm 1. However, to authors' experience, implementation of Algorithm 2 is clearly more easy.

\footnotetext{
${ }^{4}$ In [5], stability of zero-dynamics has been numerically established.

${ }^{5}$ It can be shown that $\Gamma_{N o m}$ is invertible in the physical domain of the application $[11,8]$.

${ }^{6}$ This value and the following represent parameters variations with respect to their nominal values.
} 

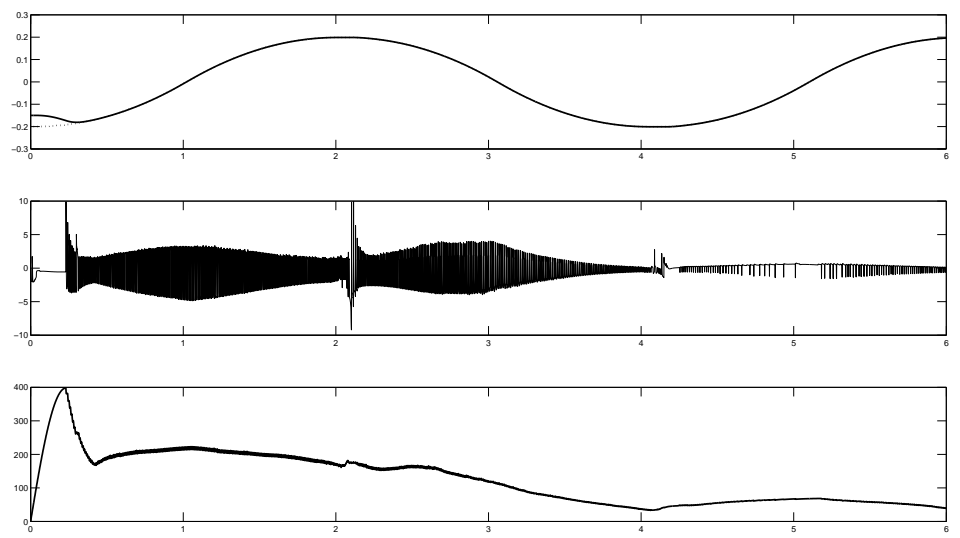

Figure 6: CONTROL ALGORITHM 1. Top. Current (solid line) and desired (dashed line) position trajectories $(m)$ versus time (sec). Center. Control input $u(V)$ versus time (sec). Bottom. Control gain $K$ versus time (sec).
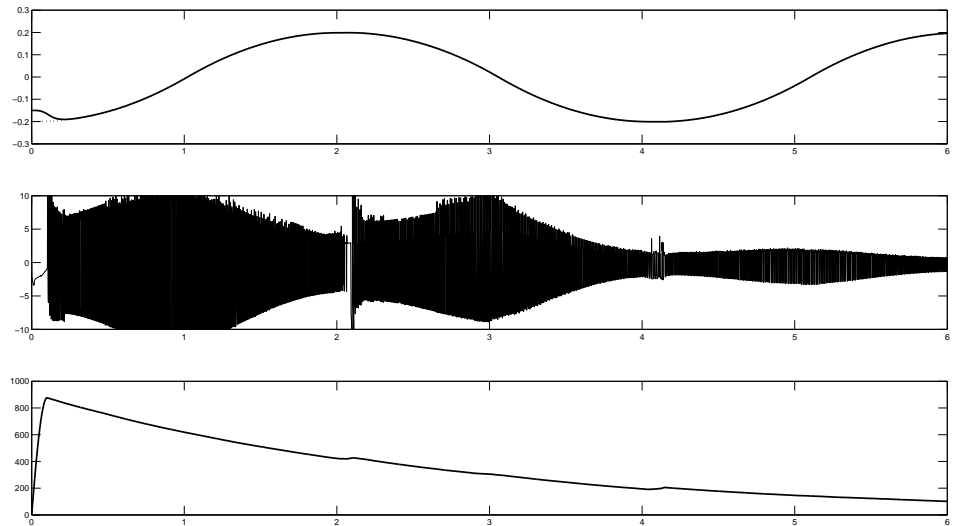

Figure 7: CONTROL ALGORITHM 2. Top. Current (solid line) and desired (dashed line) position trajectories $(m)$ versus time (sec). Center. Control input $u(V)$ versus time $(\mathrm{sec})$. Bottom. Control gain $K$ versus time (sec).

\section{Conclusion}

This paper proposes two new methodologies for adaptive sliding mode controller design. The both algorithms allow establishing of the sliding mode via the sliding mode control laws with gain adaptation without a priori knowing uncertainties/perturbations bounds while the adaptive gains values are both not over-estimated. The first algorithm is based on evaluation of uncertainties/perturbation by using equivalent control concept that requires employment of low-pass filter. The second adaptive control law does not estimate the boundary of pertur- 
bations/uncertainties and yields establishing a real sliding mode. The efficacy of these new strategies has been confirmed on a tutorial example as well as by controlling the electropneumatic actuator. Future works will concentrate on extending the developed methodologies to both MIMO uncertain nonlinear systems and to systems with higher order adaptive-gain sliding mode control, as well as on experimental validating the advanced algorithms.

\section{References}

[1] G. Bartolini, A. Ferrara, E. Usai, and V.I. Utkin, "On multi-input chattering-free secondorder sliding mode control", IEEE Trans. Autom. Control, vol.45, no.9, pp.1711-1717, 2000 .

[2] M. Belgharbi, D. Thomasset, S. Scavarda, andS. Sesmat, ÒAnalytical model oftheSSowstageofapneumatic servo-distributor for simulationandnonlinear controlÓ, Scandinavian International Conference on Fluid Power SICFP'99, Tampere, Finland, 1999.

[3] I. Boiko, and L. Fridman, "Analysis of chattering in continuous sliding-mode controllers", IEEE Transaction on Automatic Control, vol.50, no.9, pp.1442-1446, 2005.

[4] I. Boiko, L. Fridman, A. Pisano, and E. Usai, "Analysis of chattering in systems with second order sliding modes", IEEE Transactions on Automatic Control, vol.52, no.11, pp. 2085-2102, 2007.

[5] X. Brun, M. Belgharbi, S. Sesmat, D. Thomasset, and S. Scavarda, "Control of an electropneumatic actuator, comparison between some linear and nonlinear control laws", Journal of Systems and Control Engineering, vol.213, no.5, pp.387-406, 1999.

[6] R. Castro-Linares, A. Glumineau, S. Laghrouche, and F. Plestan, "High order sliding mode observer-based control", 2nd IFAC Symposium on System, Structure and Control, Oaxaca, Mexico, 2004.

[7] A.F. Filippov, "Differential equations with discontinuous right-hand side", Kluwer Academic Publishers, Dordrecht, The Netherlands, 1988.

[8] A. Girin, F. Plestan, X. Brun, and A. Glumineau, "Robust control of an electropneumatic actuator: application to an aeronautical benchmark", IEEE Transactions on Control Systems Technology, vol.17, no.3, pp.633-645, 2009.

[9] C.E. Hall, and Y.B. Shtessel, "Sliding mode disturbance observer-based control for a reusable launch vehicle", AIAA J. Guidance, Control, and Dynamics, vol.29, no.6, pp.1315-1328, 2006.

[10] Y.-J. Huang, T.-C. Kuo, and S.-H. Chang, "Adaptive sliding-mode control for nonlinear systems with uncertain parameters", IEEE Trans. Syst., Man, and Cybernetics-Part B: Cybernetics, vol.38, no.2, pp.534-539, 2008.

[11] S. Laghrouche, M. Smaoui, F. Plestan, and X. Brun, "Higher order sliding mode control based on optimal approach of an electropneumatic actuator", International Journal of Control, vol.79, no.2, pp.119-131, 2006. 
[12] S. Laghrouche, F. Plestan, and A. Glumineau, "Higher order sliding mode control based on integral sliding surface", Automatica, vol.43, no.3, pp.531-537, 2007.

[13] H. Lee, and V.I. Utkin, "Chattering suppression methods in sliding mode control systems", Annual Reviews in Control, vol.31, pp.179-188, 2007.

[14] A. Levant, "Sliding order and sliding accuracy in sliding mode control", Int. J. Control, vol.58, no.6, pp.1247-1263, 1993.

[15] A. Levant, "Universal SISO sliding-mode controllers with finite-time convergence", IEEE Trans. Autom. Control, vol.49, no.9, pp.1447-1451, 2001.

[16] A. Levant, "Principles of 2-sliding mode design", Automatica, vol.43, no.4, pp.576-586, 2007.

[17] D. Mac Cloy, "Discharge characteristics of servo valve orifices", Fluid Power International Conference, Olympia, Greece, pp.43-50, 1968.

[18] D. Munoz, and D. Sbarbaro, "An adaptative sliding mode controller for discrete nonlinear systems", IEEE Trans. Industrial Electronics, vol.47, no.3, pp.574-581, 2000.

[19] F. Plestan, A. Glumineau, and S. Laghrouche, "A new algorithm for high order sliding mode control", International Journal of Robust and Nonlinear Control, vol.18, no.4-5, pp.441-453, 2008.

[20] S. Sesmat, and S. Scavarda, "Static characteristics of a three way servovalve", Conference on Fluid Power Technology, Aachen, Germany, pp.643-652, 1996.

[21] J.L. Shearer, "Study of pneumatic processes in the continuous control of motion with compressed air", Trans. Am. Soc. Mech. Eng., vol.78, pp.233-249, 1956.

[22] J.-J. Slotine, and S.S. Sastry, "Tracking control of nonlinear system using sliding surfaces, with application to robot manipulators", Int. J. Control, vol.38, no.2, pp.465-492, 1983.

[23] J.-J. Slotine, and W. Li, Applied nonlinear control, Prentice Hall Ed., 1991.

[24] C.W. Tao, M.L. Chan, and T.T. Lee, "Adaptive fuzzy sliding mode controller for linear systems with mismatched time-varying uncertainties", IEEE Trans. Syst., Man, and Cybernetics-Part B: Cybernetics, vol.33, no.2, pp.283-294, 2003.

[25] V.I. Utkin, J. Guldner, and J. Shi, Sliding mode in control in electromechanical systems, Taylor \& Francis Ed., 1999.

\section{Appendix}

Proof of Lemma 1. Suppose that $\sigma(x, t) \neq 0$. From $K$-dynamics (8), and given that functions $\Psi$ and $\Gamma$ are supposed bounded, it follows that $K$ is increasing and there exists a time $t_{1}$ such that

$$
K\left(t_{1}\right)>\frac{\Psi_{M}}{\Gamma_{m}}
$$


Note that this gain has a finite value by the absolute continuity property of $K(t)$. From $t=t_{1}$, given $K$-dynamics, gain $K$ is large enough to make the sliding variable $\sigma$ decreasing. Then, it yields that, in a finite time $t_{2}, \sigma=0$ and $K\left(t_{2}\right)$ admits a bounded value. It yields that there always exists a positive constant $K^{*}$ such that $K^{*}>K(t)$, for all $t \geq 0$.

Proof of Lemma 2. Suppose that $|\sigma(x, t)|>\epsilon$. From $K$-dynamics, and given that functions $\Psi$ and $\Gamma$ are supposed bounded, it follows that $K$ is increasing and there exists a time $t_{1}$ (see Figure 8) such that

$$
K\left(t_{1}\right)=\left|\frac{\Psi\left(t_{1}\right)}{\Gamma\left(t_{1}\right)}\right| .
$$

From $t=t_{1}$, given $K$-dynamics, gain $K$ is large enough to make the sliding variable $\sigma$ decreasing. Then, it yields that, in a finite time $t_{2}$ (see Figure 8), $|\sigma|<\epsilon$. It yields that gain $K$ is decreasing from $t_{2}$, gain $K$ being at a maximum value at $t=t_{2}$. From $K$-dynamics, it yields that there exists a time instant $t_{3}>t_{2}$ (see Figure 8) such that

$$
K\left(t_{3}\right)=\left|\frac{\Psi\left(t_{3}\right)}{\Gamma\left(t_{3}\right)}\right| .
$$

From $t=t_{3}$, gain $K$ is not large enough to counteract perturbations and uncertainties as it is decreasing. It yields that there exists a time instant $t_{4}>t_{3}$ such that $\left|\sigma\left(t_{4}\right)\right|>\epsilon$. The process then restarts from the beginning. By the assumptions (3), the gains $K\left(t_{i}\right)$ remain bounded uniformly on $t_{i}$. In fact,

$$
K\left(t_{i}\right)=\left|\frac{\Psi\left(t_{i}\right)}{\Gamma\left(t_{i}\right)}\right| \leq \frac{\Psi_{M}}{\Gamma_{m}}:=K^{* *}
$$

and, hence, there always exists a finite constant $K^{*}$ such that

$$
K^{*} \leq K^{* *}
$$

which proves the desired result.

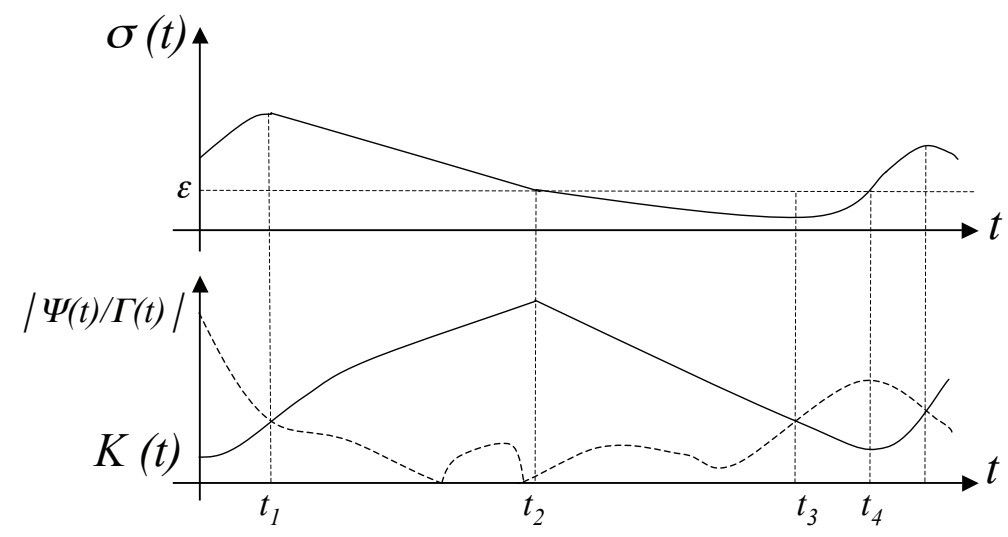

Figure 8: Scheme describing the behaviour of $\sigma$ (Top) and $K$ (Bottom) versus time. 